\title{
Las partículas elementales básicas como nucleantes de la martensita ${ }^{(\bullet)}$
}

\begin{abstract}
V.J. Aguinaco-Bravo ${ }^{(*)}$ y J. Oñoro ${ }^{(*)}$
Resumen La transformación martensítica es una transformación de fase que ocurre sin difusión y que modifica sensiblemente la microestructura y propiedades de los materiales. En este trabajo se propone que la nucleación de la transformación martensítica está causada por la existencia de unas partículas elementales básicas (basic elementary particles, bep). La formación de estas partículas elementales básicas se realizará en varias etapas. A alta temperatura se formarán vacantes, divacantes, trivacantes y, posteriormente, racimos de vacantes. Durante el enfriamiento se colapsarán generando circuitos prismáticos de dislocaciones. Cuando estas partículas alcanzan un radio crítico y cumplen con ciertas condiciones de energía elástica, las definimos como bep. Cuando estas bep presentan una orientación cristalográfica que coincide con el plano de hábito de la matriz original dará como resultado un núcleo martensítico.
\end{abstract}

Palabras clave: Transformación martensítica. Nucleación martensítica. Partículas elementales básicas.

\section{The basic elementary particles as martensitic nucleous}

\begin{abstract}
The martensitic transformation is a diffusionless structural change that produces an important modification of the microstructure and properties of materials. In this paper we propose how the martensitic phase is nucleated from a basic elementary particle (bep). The bep is formed in several stages. Vacancies, divacancies, trivacancies, etc. are formed at high temperature, which collapse into prismatic dislocation loops during the cooling process. We define a bep as a dislocation loop reaching a critical radius and fulfiling certain elastic energy conditions. A martensitic nucleus is a bep that coincides crystallographically with the habit plane of the matrix.
\end{abstract}

Keywords: Martensitic transformation. Martensitic nucleation. Basic elementary particles.

\section{INTRODUCCIÓN}

La martensita fue inicialmente identificada como una fase del acero, una microestructura que resulta de las especiales consecuencias de una transformación de fase, producida por un enfriamiento muy rápido desde la fase austenítica. La descomposición de la fase austenítica ccc en un enfriamiento lento supone su separación en dos fases, ferrita y cementita, con diferentes contenidos de carbono. El proce-

(•) Trabajo recibido el día 6 de noviembre de 1996. Nueva versión enviada por los autores el día 29 de octubre de 1998 y aprobado en su forma final el 9 de noviembre de 1998.

Este artículo es parte de la tesis doctoral presentada por Vicente Aguinaco Bravo en noviembre de 1995 al Dpto. de Ingeniería y Ciencia de Materiales, ETSI Industriales, Universidad Politécnica de Madrid (España).

(*) Dpto. de Ingeniería y Ciencia de los Materiales, ETSI Industriales. Universidad Politécnica de Madrid. Calle José Gutiérrez Abascal 2, 28006-Madrid (España). so requiere, por lo tanto, una difusión. Si el acero se enfría rápidamente, el proceso difusional no tiene lugar; sin embargo, la fuerza que activa la transformación, que es función del gradiente de temperatura, es tan grande que el acero sufrirá una transformación inducida por una deformación mediante un proceso sin difusión, obteniendo una estructura tetragonal muy cercana a cc. La acción mecánica de este tipo de transformación introduce una energía de deformación elástica y una alta densidad de dislocaciones, así como maclas y otros defectos en la red. Esto, combinado con la distorsión producida por una sobresaturación de carbono, da lugar a una estructura de elevada resistencia mecánica y por lo tanto de gran utilidad para la industria.

Sabemos que la transformación martensítica en aceros es sólo un ejemplo de las muchas transformaciones sin difusión identificadas actualmente. Los materiales susceptibles de una transformación martensítica incluyen una gran variedad de aleaciones metálicas, materiales ferro-eléctricos, 
materiales cerámicos, fases superconductoras, etc. La característica del producto martensítico difiere en cada sistema, pero todos son particularmente sensibles a una relación cristalográfica entre la martensita y la matriz.

Pondremos al lector en antecedentes sobre los estudios tradicionales respecto a la transformación martensítica para hablar a continuación de la formación de las bep. Después, profundizaremos en las transformaciones martensíticas desde el punto de vista del plano invariante de deformación, pid, discutiremos las hipótesis planteadas y en un artículo posterior se presentarán los resultados obtenidos de una simulación de una transformación martensítica.

\section{ANTECEDENTES}

Los cristales no se componen simplemente de átomos idénticos en sitios idénticos a través de una red tridimensional repetida regularmente. Contienen imperfecciones o defectos. Las imperfecciones que interrumpen la estructura son, en su mayoría, imperfecciones en la red espacial. Dado que la red espacial es un concepto geométrico, es natural clasificar las imperfecciones de la red geométricamente. Las vacantes se forman por la acción de extraer un átomo de su sitio atómico y los intersticiales por el hecho de introducir un átomo en un sitio no atómico. Se ha demostrado mediante una extensa experimentación que se pueden producir las vacantes y los defectos intersticiales en los materiales por medio de una deformación plástica y por medio de una irradiación de partículas de alta energía; además, se pueden retener estos defectos mediante un enfriamiento rápido. De esto último podemos decir que, termodinámicamente hablando, hay un número estable de vacantes. El cambio de energía libre $\Delta F$, energía de Helmholtz, está asociado con la introducción de $n$ vacantes en la red cristalina.

La velocidad a la cual las vacantes se mueven de un lugar a otro decrece exponencialmente con la disminución de la temperatura y, consecuentemente, es posible retener una alta concentración de vacantes mediante un proceso de temple (1). Cuando una vacante se encuentra con otra vacante y el espécimen está sometido a una alta temperatura, la formación de divacantes es proporcional al cuadrado de la concentración de vacantes. En 1952, Seitz (2) propuso que, durante el proceso de temple, era posible retener una alta concentración de vacantes que se precipitaban en circuitos cerrados de dislocaciones. Encontramos una confirmación sobre este fenómeno en el trabajo de Kimura (3 y 4), quien evidenció que durante el proceso de enfriamiento rápido se formaba un número considerable de divacantes precipitándose en grandes racimos de vacan- tes. Estudios posteriores (5) demostraron que núcleos martensíticos se forman a partir de vacantes durante un enfriamiento rápido. Los racimos de vacantes podían ser agujeros de forma esférica o semejando discos planos de forma elipsoidal, y tras el estudio de este último caso, se determinó que la red se relajaba alrededor del racimo formando un circuito prismático de dislocaciones (6).

El concepto de circuito cerrado de dislocaciones prismáticas lo podemos definir en función de dos vectores. La dirección de la línea de dislocación en cualquier punto dado viene descrito por el vector unitario, $t$, que es paralelo a la línea y que debe ser continuo alrededor del circuito cerrado de dislocación. El segundo vector es el de traslación $b$, normalmente llamado vector de Burgers, $b$, el vector indica qué cantidad y en que dirección ha cambiado el deslizamiento del plano en la red respecto a la red original. El vector de Burgers es exactamente el mismo en cualquier punto del circuito mientras que el vector $t$ cambia de dirección continuamente. Si se inserta un área de vacantes en un lugar donde se ha quitado una parte de plano atómico puede formarse una dislocación prismática de signo opuesto.

Resultados experimentales han sugerido que hay al menos tres tipos diferentes de sumideros de vacantes dependiendo de su origen. En un primer lugar tenemos los que se forman en las superficies y los que se forman en las juntas de grano, los cuales teóricamente pueden absorber todas las vacantes que no están en equilibro, en segundo lugar está la formación de agujeros esféricos sobre dislocaciones, y hay también otro mecanismo que puede generar sumideros de vacantes: las mismas vacantes, al formar racimos se colapsarán para formar circuitos cerrados de dislocaciones (7).

Schoeck (8) y Bauerle (1), quien lo demostró más tarde experimentalmente, confirmaron que el tiempo que una vacante sobrevive como vacante sencilla puede considerarse como inversamente proporcional a la concentración de vacantes sencillas, de modo que mientras más alta sea la temperatura del proceso de temple, más corto será el tiempo promedio que una vacante exista como vacante sencilla. Con esto podemos decir que las imperfecciones de la red más abundante que emigrarán serán las divacantes, que se moverán para formar grandes racimos de vacantes que se colapsarán en circuitos cerrados de dislocaciones.

Khachaturyan (9) desarrolló un tratamiento matemático para calcular la energía elástica asociada a una inclusión martensítica en un medio anisotrópico, en función de la forma y del plano de hábito. La teoría general de la energía de deformación fue propuesta en 1966 por Khachaturyan (9 y 10), quien formuló la ecuación exacta de la energía de deformación y el desplazamiento elástico en términos de la transformada de Fourier, considerando 
una mezcla coherente de dos fases pasando por un acomodo estructural arbitrario y con inclusiones de características elásticas anisotrópicas. La técnica consiste en calcular la orientación del plano de hábito que depende de la relación axial con la estructura del precipitado y la estructura de la fase original.

La teoría aquí presentada de la existencia de las bep como elementos nucleantes de la transformación martensítica nos permitirá calcular la energía de deformación de una distribución arbitraria de inclusiones coherentes. También, por medio de ella plantearemos un modelo que represente la energía de deformación de la transformación martensítica conforme esta avanza, identificando las etapas más favorables con respecto a la energía de deformación. Estos racimos de vacantes se colapsarán para formar circuitos cerrados de dislocaciones que tendrán la peculiaridad de tener una forma elipsoidal.

Proponemos que cuando se han precipitado los racimos de vacantes en circuitos cerrados de dislocaciones, estos llegan a coincidir cristalográficamente con la estructura de la matriz creando un plano que no ha sufrido ningún cambio en su orientación y sólo ha experimentado una rotación de cuerpo rígido, recordando que la transformación martensítica garantiza un plano invariante de deformación (11). La extensión de esa discontinuidad puede decrecer su energía de deformación alterando su forma, su interfase y el plano de hábito, convirtiendo la deformación en un plano invariante de deformación. Este ajuste morfológico se facilita por la participación adicional de dislocaciones que a menudo conducen a relaciones cristalográficas que son identificadas como una transformación martensítica.

\section{LA PARTÍCULA ELEMENTAL BÁSICA, $B E P$}

Como ya hemos expuesto, la presencia de racimos de vacantes provocará la existencia de un campo elástico que estará restringido por la matriz original. Basándonos en el modelo de Eshelby (12) podemos suponer que la partícula que está en un medio infinito, homogéneo e isotrópico, estará sometida a un estado de transformación donde la matriz impondrá unas restricciones homogéneas de deformación sobre la partícula.

El estado elástico lo determinamos utilizando el modelo de Eshelby mediante una sucesión de pasos imaginarios; se supondrá que la morfología de la bep tiene una forma elipsoidal y por la razón de que esta forma tiene la propiedad de hacer que la deformación dentro de la partícula sea uniforme.

Ahora bien, si la concentración de vacantes en un sólido excede el equilibrio térmico, existe una tendencia de la vacante a condensarse en grandes agregados en forma de racimos. Se presume que la formación de los anillos de dislocaciones se precipita a partir de discos de vacantes cuando han alcanzado un tamaño crítico (6). Una vez alcanzada esta etapa se podrá calcular la supersaturación necesaria para mantener los anillos de dislocaciones creciendo mediante el mecanismo de ascenso. Partimos de un dato cierto, y es que la supersaturación debe ser igual al valor de la concentración de vacantes. Además, como todas las dislocaciones formadas por una condensación de vacantes deben de pasar por unas etapas iniciales de crecimiento, obtenemos de esta manera el límite inferior necesario para una supersaturación de vacantes.

Basándose en la teoría recopilada hasta aquí se desea señalar que, aunque esté presente un estado de deformación, como confirma la teoría tradicional, se propone considerar estos discos de dislocaciones prismáticas como partículas elementales en la transformación martensítica.

Partimos de la condición de que estas partículas tienen una interfase coherente con la matriz original para que la deformación sea homogénea y así forme una elipse cerrada de dislocaciones parciales. Como la deformación elástica dentro de una partícula de forma elipsoidal (vector de deformación) es siempre homogénea y es el caso más común cuando las interfases coherentes se encuentran afectadas por fuerzas de origen elástico, el punto de partida es correcto. Por tanto, tenemos la presencia de una partícula que debe ser evaluada energéticamente con respecto a la energía de deformación elástica.

La nueva partícula coherente ceñida a ese estado tendrá una red cristalina diferente a la de una partícula que está bajo un estado libre de esfuerzos. Esta diferencia se debe a la deformación elástica causada por el desajuste de las fronteras de las interfases. Cualquier partícula coherente pasará por una deformación elástica homogénea que restablecerá el plano de hábito al estado que tenía antes de que ocurriera la transformación para asegurar que el plano de hábito de la nueva fase y la matriz coincidan sin distorsión. La simple combinación de la deformación, debida a la transformación y libre de esfuerzos, $\varepsilon_{\mathrm{ij}}^{0}$, y la deformación elástica en la partícula resulta en la coincidencia del plano de hábito del cristal antes y después de la transformación. La deformación total en la partícula es por definición un plano invariante de deformación donde el plano de hábito viene a ser un plano invariante. El estudio de este fenómeno lo haremos desde una descripción matemática en términos de la correspondiente función de Green, $G$, que matemáticamente es la solución del mismo operador diferencial con las mismas condiciones de contorno (13).

De acuerdo con la ecuación general del plano invariante $u_{\mathrm{i}}=\varepsilon l_{\mathrm{i}} n_{\mathrm{j}} r_{\mathrm{j}}$, donde $u$ representa el desplazamiento de todos los puntos sobre el plano en la 
dirección del vector unitario, $l$ y $\varepsilon$ es una constante adimensional que describe la cantidad de deformación, un plano invariante de deformación está descrito por el producto de dos vectores, de los cuales uno es $n_{0}$, normal al plano invariante, y el otro vector cortante $S=\varepsilon l$. De tal manera que la deformación homogénea de la partícula coherente puede ser descrita por $u_{i j}=S_{\mathrm{i}} n_{\mathrm{j}}^{0}$, de modo que podemos representar el esfuerzo cortante $S$ por medio de la función de Green.

$$
S=\hat{\Omega}\left(n_{0}\right) \hat{\sigma}^{0} n_{0}
$$

donde $\widehat{\Omega}\left(n_{0}\right)$ es el tensor de la matriz elástica y $\hat{\sigma}$ es el tensor del esfuerzo de transformación. La matriz del plano invariante está definida de la siguiente forma

$$
\hat{A}=\hat{I}+S\left(n_{0}\right) * n_{0}
$$

Cualquier traslación de $r$ de la fase original se transformará en $r^{\prime}$ después de la transformación de fase

$$
\begin{gathered}
r^{\prime}=\hat{A} r=\left(\hat{I}+S\left(n_{0}\right) * n_{0}\right) r \\
=r+S\left(n_{0}\right)\left(n_{0} r\right)
\end{gathered}
$$

La ecuación anterior determina la relación de orientación entre la red cristalina de la fase original y la partícula. También nos dice que todas las traslaciones de la red cristalina que estén en el plano de hábito permanecerán inalterables por el reajuste de la misma. Una vez determinado $r^{\prime}$ sabremos si la orientación de la partícula ha coincidido con el plano invariante de deformación, lo que nos asegura que la transformación martensítica se ha llevado a cabo. Mediante las ecuaciones desarrolladas por Khachaturyan (9) y por García Moliner (13) podemos determinar el estado energético de la partícula y predecir si la transformación tiene lugar. Por otro lado, minimizando la función $B(n)$, esta función contiene toda la información sobre las propiedades elásticas y cristalográficas del sistema y la transformación de fase- aseguramos la coincidencia del plano de hábito con el plano invariante de deformación. Así, se cumple nuestro propósito de validar el modelo propuesto de nucleación de la martensita, que queda justificado por la teoría de la deformación elástica.

Como ya hemos mencionado anteriormente, el exceso de vacantes está presente en el material cuando, partiendo de un enfriamiento rápido, estas se precipitan sobre planos compactos para formar discos. Cuando esos discos son lo suficientemente grandes, serán energéticamente favorables para que se colapsen formando circuitos cerrados de disloca- ciones. La longitud de la partícula la calcularemos por medio de la energía superficial, y para tal efecto se parte de que tiene un radio crítico $r_{\mathrm{c}}$ que depende del calor desprendido durante su formación. De tal forma que conociendo la energía superficial de la aleación calcularemos su radio crítico, ya que guarda una proporción de aproximadamente tres veces el cociente de la energía superficial entre el calor desprendido. Esta aproximación resulta estar dentro de los valores esperados, dado que usualmente un disco formado por circuitos cerrados de dislocaciones suele tener un tamaño mucho mayor. Con esta suposición garantizamos que la partícula elemental sea lo suficientemente pequeña como para iniciar una nucleación de la martensita cumpliendo con los requisitos de identidad cristalográfica de la fase martensítica.

Si consideramos el hecho de que las vacantes existen en un medio semiinfinito contenidas en un espacio $z \geq 0$ y que tenemos las siguientes condiciones de frontera, a saber, $+\infty$ y $z=0$, asumimos que los esfuerzos elásticos transmitidos por la superficie libre; que está en $z=0$, son nulos. Por analogía, si el campo electromagnético existe (14), se le pueden aplicar las leyes electromagnéticas, es decir que los componentes tangenciales de los campos eléctrico y magnético son continuos en $\mathrm{z}= \pm 0$. En una interfase entre dos medios dieléctricos en una frontera cuasiestática, la forma de conectar las dos superficies consistiría en imponer condiciones de continuidad entre el potencial electrostático y la componente normal del vector de desplazamiento eléctrico. La justificación matemática puede basarse en términos de la función de Green, $G$, que proporciona la solución del operador diferencial con las mismas condiciones de frontera. La interpretación física de esta solución es que la función de Green permite la propagación de las fluctuaciones básicas de un espacio a otro. Basándose en el método desarrollado por García Moliner (13), Surface Function Green Matching, SFGM, es posible estudiar la unión entre dos vacantes. Estas vacantes se convierten en divacantes para formar después racimos de vacantes que cumplen las condiciones de frontera establecidas.

La formación de vacantes, divacantes, trivacantes y así sucesivamente hasta formar racimos de vacantes fue probado por Kimura ( 3 y 4 ), quien demostró que estos racimos se colapsaban en circuitos cerrados de dislocaciones durante el enfriamiento.

Una vez que un circuito cerrado de dislocaciones cumple con la condición de radio crítico y ciertas condiciones de energía elástica, puede seguir creciendo dependiendo de la temperatura, teniendo una interfase coherente. Siguiendo la misma línea de razonamiento se puede decir que para que esta partícula sea una bep deberá de poseer una energía 
elástica del tipo $B\left(n_{0}\right)$ mínima, de tal forma que cuando alcance su valor mínimo sabremos que ha coincidido con el plano invariante, condición necesaria y suficiente para asegurar que se puede llevar a cabo una transformación martensítica. Podemos suponer que la partícula tiene las mismas propiedades que la matriz. Con base a lo expuesto anteriormente decimos que la energía de deformación elástica de una bep será

$$
\begin{gathered}
E_{\text {bep }}=\frac{1}{2} \int \frac{d^{3} k}{(2 \pi)^{3}} B(n)|\theta(k)|^{2} \geq \frac{1}{2} \min B(n) \\
\int \frac{d^{3} k}{(2 \pi)^{3}}|\theta(k)|^{2}
\end{gathered}
$$

donde $E_{\mathrm{bep}}$ es la energía elástica de deformación de una bep, $B(n)$ es la función que representa las propiedades del sistema, y $\theta(k)$ es la transformada de Fourier de la función de forma de la partícula y corresponde con la función de interferencia de Laue, donde $k$ es la distancia de un punto en la red recíproca en el espacio recíproco.

\section{DISCUSIÓN}

El proceso de transformación martensítica produce una transformación de fase que habitualmente aumenta las propiedades de resistencia de un material. Algunos autores han desarrollado modelos que relacionan los sumideros de vacantes como parte del proceso de endurecimiento (15), mientras que otros han propuesto mecanismos que consideran los saltos de dislocaciones o la formación de agujeros esféricos sobre las dislocaciones (16). Por otro lado, también se ha dicho que las partículas elementales martensíticas son generadas a partir de vacantes que se han creado durante un enfriamiento rápido (5). Pero hasta donde se ha podido comprobar, no se ha considerado la energía de deformación como una parte importante en la precipitación de los circuitos prismáticos de dislocaciones creados a partir de racimos de vacantes ni la técnica de $S F G M$ se ha empleado para el estudio de la formación de racimos de vacantes.

El concepto fenomenológico de la teoría de la transformación de la martensita dice que "el plano invariante debe de ser el plano de hábito de un cristal martensítico cumpliendo con la condición de energía mínima de deformación".

La función $B(n)$ que representa las propiedades del sistema en la dirección de un vector unitario $n=k /|k|$

$$
B(n)=\lambda_{\mathrm{ijkl}} \varepsilon_{\mathrm{ij}}^{0} \varepsilon_{\mathrm{k} 1}^{0}-n_{\mathrm{i}}^{0} \sigma_{\mathrm{ij}}^{0} \Omega_{\mathrm{jl}}(n) \sigma_{\mathrm{lm}}^{0} n_{\mathrm{m}}
$$

donde $\lambda_{\mathrm{ijkl}}$ es una constante elástica, $\sigma_{\mathrm{ij}}^{0}=\lambda_{\mathrm{ijk}} \varepsilon_{\mathrm{ij}}^{0}, \varepsilon_{\mathrm{ij}}^{0}$ es la deformación debida a una transformación bajo un estado libre de esfuerzos, y $\Omega_{\mathrm{jl}}(n)$ es el tensor inverso de $\Omega^{-1}{ }_{\mathrm{ij}}(n)=\lambda_{\mathrm{ijk}} n_{\mathrm{k}} n_{\mathrm{l}}$.

Suponiendo que $B(n) \geq 0$ existirá una dirección $n=n_{0}$ que dé un valor mínimo a la función $B(n)$. En otras palabras, en el caso de una deformación en el plano invariante representará una transformación de deformación libre de esfuerzo, y la ecuación de la energía elástica de deformación tendrá entonces la siguiente forma

$$
E_{\text {bep }}=\frac{1}{2}[\min B(n)] V=\frac{1}{2} B\left(n_{0}\right) V \rightarrow 0
$$

donde $V$ es el volumen de la partícula en estudio.

Esto se deduce de la condición de energía mínima de deformación donde el vector normal al plano invariante es también un plano normal al plano de hábito; dicho de otra forma: "el plano invariante siempre coincide con el plano de hábito". De esta manera se puede decir que una placa que se encuentra en un pid tiene una energía elástica mínima muy próxima o igual a cero. Esto supone que el plano de hábito de la transformación martensítica es un plano invariante de la transformación, tesis apoyada por la teoría elástica al confirmar que el plano invariante asegura un mínimo de energía elástica

\section{CONCLUSIONES}

- Se ha presentado la hipótesis de la formación de una partícula elemental bep, a partir de la formación de vacantes simples a elevada temperatura, que al unirse dan lugar a divacantes, trivacantes y así sucesivamente hasta que se colapsan formando racimos de vacantes, los que a su vez transforman en circuitos cerrados de dislocaciones de forma elipsoidal.

- Si los circuitos cerrados de dislocaciones cumplen con la condición de energía elástica mínima, entonces el plano de hábito de la partícula bep coincide con el plano de la fase original creándose un plano invariante de deformación, condición necesaria y suficiente para que exista una transformación martensítica.

- Minimizando la función $B(n)$ de la energía elástica es posible predecir que tendrá lugar una transformación martensítica.

- La hipótesis presentada plantea la teoría de una nucleación a partir de una energía de deformación. Una bep o inclusión coherente, al sufrir una deformación elástica homogénea y cumpliendo con la condición de mínima energía, restablecerá el plano de hábito al estado que tenía antes de que ocurriera la transformación 
garantizando una coincidencia exacta del plano de hábito sin distorsión de la nueva fase y la matriz.

\section{REFERENCIAS}

(1) Bauerle, J.E. y Koehler, J.S. Phys. Rev., 107, 1957: 1499.

(2) SEITZ, F. Adv. Phys., 1, 1952: 43.

(3) Kimura, H., Maddin. R. y Kuhlmann-Wilsdorf, D. Acta Metallurgica, 7, 1959: 145.

(4) Kimura, H., Maddin. R. y Kuhlmann-Wilsdorf, D. Acta Metallurgica, 7, 1959: 154.

(5) Sadananda, K. y Marcinkowski, M.J. Acta Metallurgica, 22, 1974: 863.

(6) Hull, Derek. Introduction to Dislocations. Materials Science and Technology. Pergamon Press, $2^{\mathrm{a}}$ ed., 1979.

(7) Tartour, P. y Washburn, T. Phil. Mag., 18, 1868: 1257.

(8) Schoeck, G. y Tiller, W.A. Phil. Mag., 5, 1960: 43.
(9) Khachaturyan, A.G. Sov. Phys. Solid State, 8, 1967: 2163.

(10) Khachaturyan, A.G. Theory of Structural Transformation in Solids. John Wiley \& Sons, $1^{\text {a }}$ ed., 1983.

(11) Wayman, C.M. Introduction to the Crystallography of Martensitic Transformation. Materials Science Series. MacMillan, $1^{\text {a }}$ ed., 1964.

(12) Eshelby, J.D. Proc. Roy. Soc., A241, 1957: 376.

(13) Garcia Moliner, F. y Velasco, V.R. Theory of Single and Multiple Interfaces: The Method of Surface Green Function Matching. World Scientific Publishing Co, Singapur, $1^{\text {a }}$ ed., 1992.

(14) Nabarro, F.R.N. Theory of Crystal Dislocations. International Series of Monographs on Physics. Oxford at the Calderon Press, $1^{\text {a }}$ ed., 1967.

(15) Maddin, R. y Cottrell, A.H. Phi. Mag., 46, 1955: 735.

(16) Friedel, J. Les Dislocation. Gauthier-Villars, París, $1^{\mathrm{a}}$ ed., 1956. 